Chirurg 2012 $\cdot 83: 773-774$

DOI 10.1007/s00104-012-2291-0

Online publiziert: 1. September 2012

๑) Springer-Verlag 2012

\section{U. Settmacher}

Klinik für Allgemein-, Viszeral- und Gefäßchirurgie, Universitätsklinikum Jena

\title{
Arteriovenöse Zugänge für die chronische Hämodialyse
}

Die terminale Niereninsuffizienz wird in der modernen Medizin bereits seit längerer Zeit erfolgreich mit Peritonealdialyse, Hämodialyse oder bestenfalls mit einer allogenen Nierentransplantation behandelt. Damit kann für die Patienten trotz des Versagens der eigenen Nieren ein langes Überleben gewährleistet werden. Ein großer Anteil dieser chronischen Behandlungen wird ambulant in nephrologischen Praxen durchgeführt. Der Anspruch der Patienten ist neben einer effektiven Therapie eine hohe Lebensqualität. Um diesen beiden Anforderungen zu genügen, ist eine enge Zusammenarbeit zwischen Nephrologen und Chirurgen und ggf. Radiologen, die den Dialysezugang anlegen und eventuell auftretende Probleme der Funktionalität behandeln, zu pflegen.

Die Zahl der Patienten an der Hämodialyse wird in Deutschland auf etwa 80.000 geschätzt - dies ist somit eine große Klientel chronisch Kranker. Das Alter der Patienten an der Hämodialyse steigt ähnlich wie der Altersdurchschnitt der Gesamtbevölkerung stetig an. Die zur Niereninsuffizienz führende Grunderkrankung, Begleiterkrankungen und Stoffwechselstörungen durch die Niereninsuffizienz sind bei der Anlage und Pflege von Dialysezugängen unbedingt zu berücksichtigen. Besondere Beachtung müssen in diesem Zusammenhang Veränderungen der Haut, Gerinnungsstörungen, Eigenschaften der Anschlussgefäße für den Dialysezugang sowie die Hämodynamik lokal und die Herz-Kreislauf-Situation insgesamt finden.
Wenn man sich in dieses Gebiet der interdisziplinären Medizin begibt, sollte man unbedingt zwei Grundsätze beherzigen, die mein verehrter chirurgischer Lehrer Hans Scholz in Lehrbüchern zum Thema hinterlegt hat:

- Die Anlage eines AV-Gefäßanschlusses ist niemals eine Operation für Anfänger.

- Die Schaffung eines chronischen Gefäßanschlusses für die Hämodialyse ist immer möglich.

Im ersten Beitrag beschreiben Frau Ott und Frau Sperschneider die Anforderungen an den "guten Dialysezugang“. Der AV-Gefäßzugang ist die „Lebensader“ des Patienten. Für die suffiziente und langfristige Funktion muss daher der richtige Zugang gewählt werden. Die Anlage sollte rechtzeitig vor Dialysebeginn erfolgen. In die Pflege müssen der Patient und das Dialysepersonal eng einbezogen werden, um Komplikationen frühzeitig zu erkennen. Komplikationen am Gefäßzugang sind ein häufiger Hospitalisierungsgrund und beeinflussen die Mortalität dieser $\mathrm{Pa}$ tienten. Wegen der hohen Infektionsgefahr bei der Dialyse über einen zentralvenösen Katheter ist die rechtzeitige Anlage einer Dialysefistel für die Morbidität und Mortalität zu Dialysebeginn entscheidend. Zum Zeitpunkt des Beginns der Nierenersatztherapie ist auch die kardiovaskuläre Mortalität der Patienten besonders hoch. Eine zusätzliche systemische Infektion kann rasch zum Tod führen. Wegen der Multimorbidität der Pa- tienten sollten Fehlversuche durch eine gute Planung des ersten Eingriffs minimiert werden.

Veränderungen am Dialysezugang erkennt der Nephrologe neben dem veränderten Verhalten während der Dialyse, bei der lokalen klinischen Untersuchung oder in der Duplexsonographie. Die Patienten sind angehalten, ihre Dialysefistel täglich zu prüfen und sich bei Bemerken eines Verschlusses sofort vorzustellen. Leider werden die meisten Verschlüsse erst durch das Dialysepersonal direkt vor Beginn der Behandlung bemerkt, da die chronisch kranken Patienten ausgeprägte Verdrängungstendenzen entwickeln. Immer wieder diskutiert wird die Gabe einer Thrombozytenaggregationshemmung bzw. Antikoagulation. Dies verbessert die Langzeitfunktionalität des Zugangs. Systematische Studien zum Thema fehlen allerdings.

Der Beschreibung der Anlage von nativen $\mathrm{AV}$-Zugängen widmen sich die Herren Schmitz-Rixen und Kollegen. Dem Konzept „fistula first“ wird dafür in Deutschland voll Rechnung getragen. Dies sichert den Patienten Dialysezugänge mit hohen Funktions- und niedrigen Komplikationsraten. Prinzipien die vor Anlage der AV-Fistel für jeden Patienten individuell beachtet werden müssen, werden zunächst vorgestellt. Dann werden viele wichtige Details wie die präoperative Untersuchung, die Auswahl der geeigneten Gefäße bis hin zu technischen Details der Anlage etc. beschrieben. Die Empfehlung, dass jeder Patient mit einer 
Beschreibung des neu angelegten Dialysezugangs beim Nephrologen vorgestellt werden soll, kann nur unterstützt werden.

Bei ungeeigneten oder verbrauchten oberflächlichen Armvenen bestehen vielfältige Möglichkeiten eines permanenten Dialysezugangs durch die Anlage von Prothesenshunts, welche getunnelten Vorhofkathetern prinzipiell immer vorzuziehen sind. Wann, welche Interponate anzulegen sind, beschreiben Zanow et al. in einem weiteren Beitrag. Dabei wird auf viele chirurgische Detailfragen eingegangen. Auch bei höherer Komplikationsrate als bei AV-Fisteln haben diese Dialysezugänge eine erhebliche Bedeutung als alternativer Zugang für die Hämodialyse, wenn eine AV-Fistel beim Patienten nicht mehr angelegt werden kann. Es werden zunächst die gängigen alloplastischen Materialien und neue materialtechnische Entwicklungen bzw. Modifikationen der Konfiguration von AV-Interponaten vorgestellt. Extrem wichtig bei diesen Zugängen der 2. Wahl ist die Indikationsstellung. Hier zeigt sich, dass neben einer guten Absprache mit dem Nephrologen auch die Erfahrung des Chirurgen von Bedeutung ist. In der technischen Durchführung stellen das schonende, atraumatische Operieren angefangen von der Durchführung des Eingriffs nach Möglichkeit in Lokal- bzw. Regionalanästhesie bis hin zur Vermeidung von Perigraftreaktionen den Schlüssel zum Erfolg dar. Man unterscheidet periphere von zentralen Shunts, da schon die Indikationsstellung zur Anlage differiert.

Auf das rechtzeitige Erkennen und die adäquate Therapie von Komplikationen an AV-Gefäßzugängen gehen $\mathrm{H}$. Scholz et al. ein. Zunächst werden die pathophysiologischen Mechanismen erörtert. Sowohl akute, meist operationstechnisch bedingte Komplikationen als auch chronische, durch die Nutzung des Zugangs in der Dialyse entstandene Probleme werden vorgestellt. Dabei handelt es sich lokal um morphologische Veränderungen am AV-Gefäß oder an der speisenden Arterie oder am drainierenden zentralen Venensegment oder um systemische Probleme, die entstehen können. Nervale Läsionen, Perigraftreaktionen, das Lymphödem, Infektionen periphere Minderperfusionen, venöse Stauungen (inkl. des Syndroms der retrograden venösen Arterialisation) und die kardiale Überlastung durch den hohen Shuntfluss werden diskutiert. Das Fazit: Die Mehrzahl der Komplikationen deutet sich langfristig an. Eine frühzeitige Behandlung der Ursachen kann das Eintreten einer Komplikation vermeiden bzw. hinauszögern und damit die Funktionsdauer des bestehenden Gefäßanschlusses verlängern. Ein kontinuierliches Monitoring des Gefäßanschlusses in enger Zusammenarbeit zwischen Nephrologen und Gefäßchirurgen ist die Voraussetzung und sollte dabei unbedingt beherzigt werden.

Der letzte Beitrag des Themenheftes von M. Hollenbeck und Kollegen widmet sich dem Stellenwert, der Technik der Implantation und den Komplikationsmöglichkeiten zentralvenöser Katheter als Zugänge für die Hämodialyse, die für Akutdialysen als schneller großlumiger Zugang unverzichtbar sind. Der Anteil der Dialysepatienten, die über getunnelte Katheter mit Cuff auf Dauer dialysiert werden, ist in den letzten Jahren in Deutschland rapide angestiegen und beträgt derzeit ca. $20 \%$. Dies liegt sicherlich an dem deutlich steigenden Alter unserer Patienten und der höheren Komorbidität. Diese Patienten haben aber deutlich mehr Infektionen und eine höhere Mortalität an der Dialyse als Patienten mit einer AV-Fistel oder einem AV-Shunt. Die deutschen Dialysezugangsempfehlungen geben die Indikation zur dauerhaften Dialyse über einem getunnelten Katheter „als letzte Möglichkeit, falls kein anderer permanenter $\mathrm{Zu}$ gang oder keine Peritonealdialyse möglich ist". Dies sollte bei jedem Patienten bedacht werden.

Es ist meines Erachtens gelungen, für dieses Themenheft wieder anerkannte Spezialisten des Fachs zu gewinnen, die die entsprechenden Themen umfassend und übersichtlich aktuell aufgearbeitet haben.

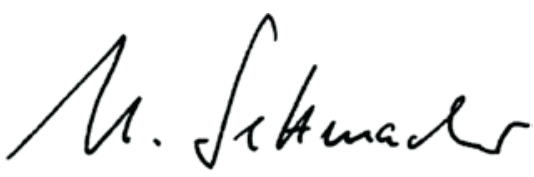

Prof. Dr. U. Settmacher

\section{Korrespondenzadresse}

Prof. Dr. U. Settmacher

Klinik für Allgemein-, Viszeralund Gefäßchirurgie, Universitätsklinikum Jena, Erlanger Allee 101, 07740 Jena utz.settmacher@med.uni-jena.de 